\title{
Adaptive forgetting in animals
}

\author{
PHILIPP J. KRAEMER and JONATHAN M. GOLDING \\ University of Kentucky, Lexington, Kentucky
}

\begin{abstract}
An argument is presented for the possibility that some cases of forgetting in animals are adaptive. In contrast to research on human memory, the idea of adaptive forgetting has not received much attention in research with animals. This paper reviews the status of adaptive forgetting in humans and then outlines an argument for adaptive forgetting in animals. The discussion includes theoretical presuppositions concerning forgetting, a review of selective phenomena indicative of adaptive forgetting in animals, a description of a possible mechanism (retrievability) for this kind of forgetting, and examination of the implications of this analysis for psychological and neurobiological approaches to memory processing.
\end{abstract}

Interest in animal memory has grown considerably over the past two decades. This trend reflects both the cognitive Zeitgeist in psychology and the growing reliance on animal models to study basic and applied issues related to brain function. As the quantity of this research has increased, so too has its complexity. What remains constant, however, is that all research on animal memory depends on one fact: forgetting. If organisms did not forget, if retention interval had no impact on behavior, then research on the types of complex questions now being pursued would be moot. Thus, the need to explain forgetting remains the core challenge for those interested in animal memory.

It is important to recognize that a successful response to that challenge will involve more than data collection and hypothesis testing; the conceptual foundation guiding the research agenda will also matter. Progress in understanding memory processing will be shaped and constrained as much by dominant metatheoretical assumptions as by empirical evidence (Bechtel, 1988). The way in which data are interpreted, the kinds of questions that are raised, and the structure of theoretical explanations will all reflect the prevailing conceptual framework within which the research is organized. An apposite expression of this principle is Maslow's (1966) aphorism, "it is tempting, if the only tool you have is a hammer, to treat everything as if it were a nail" (pp. 14-15).

With respect to animal memory, our "hammer" has been the assumption that forgetting constitutes a failed process. Although various hypotheses have been offered to explain forgetting, the alternatives share the assumption that retention deficits entail processing failures. This assumption is most often tacit, yet it is conspicuously apparent in the labels given to the theoretical alternatives: trace decay, retrieval failure, interference theory. All of these approaches seek to explain forgetting by uncover-

Correspondence should be addressed to P. J. Kraemer, Department of Psychology, University of Kentucky, Lexington, KY : $; 0506-0044$ (email: pjkrae00@pop.uky.edu). ing the source of the processing failure. This idea is certainly plausible, but the important question is whether all instances of animal forgetting constitute processing failures. Is it possible that organisms sometimes forget in the absence of a failed process? If the answer is yes, then theoretical formulations of animal forgetting will need to incorporate this functional distinction. Equally important, it will be necessary to consider seriously the implication that some forms of forgetting in animals are adaptive.

The notion of adaptive forgetting in animals might be difficult to accept, in part, because of attitudes toward our own forgetting. When we forget, the consequences are usually undesirable, frustrating, and unprofitable. It is only reasonable to assume, therefore, that forgetting serves no useful purpose and that, when we forget, it is because something has gone wrong. It seems even more difficult to see how forgetting expressed by other animals, which we assume face far less complex cognitive challenges than do we humans, could in anyway be beneficial. The purpose of what follows, however, is to present just such an argument. Two related propositions will be presented: (1) not all animal forgetting is based on a failed process, and (2) instances of forgetting that do not entail a failed process reflect an adaptive mechanism designed to enhance behavioral plasticity.

The general idea of adaptive forgetting is not unprecedented. This construct has a long history in the analysis of human memory, and it has generated considerable attention recently. MacLeod (1998) notes several historical examples, among them Ribot (1882, p. 61), who stated that "Forgetfulness, except in certain cases, is not a disease of memory, but a condition of its health and life." William James (1890, p. 680) also expressed an affinity for adaptive forgetting when he stated, "If we remembered everything, we should on most occasions be as ill off as if we remembered nothing." Perhaps the most well known early advocate of adaptive forgetting is Freud. A cornerstone of Freud's (1920/1962) psychodynamic theory was the concept of repression. He argued that people forget episodes that either include adverse emotional attributes or induce negative emotions when remembered. 
By repressing a memory, one gains some immediate psychological advantage, even if the long-term consequences of repression are deleterious. Freyd (1996), in the context of betrayal trauma theory, has recently extended the principle of repression by emphasizing the social utility of forgetting. That is, she argues that children who are sexually abused by those to whom they have strong attachment are more inclined to forget the abuse in order to function within this essential relationship. The problem with the repression concept, however, is that it has yet to gain solid empirical support (APA Report, 1995).

For those interested in animal memory, neither quaint philosophical speculation nor formulations of clinical repression will serve as strong enticements to consider the possibility that animals also display adaptive forgetting, especially given the controversial status of the latter (Loftus, 1993). Fortunately, there are other precedents for adaptive forgetting to be found in the study of human memory. One area of inquiry that is especially pertinent is the analysis of motivated or intentional forgetting, which has been studied in detail through the phenomenon of directed forgetting (R. A. Bjork, 1972, 1998; Epstein, 1972; Golding \& Long, 1998; Johnson, 1994; MacLeod, 1998). In this paradigm, explicit cues are used to designate items as "to be forgotten" or "to be remembered" (typically) after a single item or a list of items is presented. Participants are subsequently tested for their memory of both the to-be-forgotten and to-beremembered words. This research has shown that the instructions to forget (in both item and list cuing conditions) lead to a decrease in recall of the to-be-forgotten information combined with an increase in recall for tobe-remembered information (i.e., the directed forgetting effect). Originally, directed forgetting was primarily viewed as the result of encoding failure (see R. A. Bjork, 1972). More recent conceptualizations of this phenomenon, however, discuss encoding failure as leading to directed forgetting only in specific contexts (e.g., item-byitem cuing; see Basden, Basden, \& Gargano, 1993). In other contexts (e.g., list cuing), retrieval inhibition has been used to explain directed forgetting.

This approach connects directly to the idea of adaptive forgetting in that it explicitly assumes that participants can benefit by forgetting. The general idea is that, in some contexts, to-be-forgotten items are inhibited or suppressed during retrieval, unlike to-be-remembered items. One consequence of retrieval inhibition is that there is less interference to disrupt memory for to-beremembered items. According to R. A. Bjork (1989), retrieval inhibition provides "a means to suppress, set aside, destroy, or discriminate out-of-date information in memory in order to remember current information effectively" (p. 322). One source of support for the retrieval inhibition explanation is evidence that directed forgetting is not dependent on rehearsal. For example, Geiselman, R. A. Bjork, and Fishman (1983) found evidence for directed forgetting for both to-be-learned and to-be- rated words. Such a finding for the latter words was critical, because it was assumed that the to-be-rated words were not rehearsed (i.e., participants did not know that they would be required to recall them). Therefore, it was concluded that these words were inhibited during recall by a "process that blocks or inhibits access routes" (Geiselman et al., 1983, p. 70) to the to-be-forgotten words. A second source of support for retrieval inhibition are results from studies that have used retesting. In Geiselman and Bagheri (1985), subjects displayed the typical directed forgetting effect after initial testing. Then, the same words were presented to subjects, but they were all to be remembered. The results showed that recall of the words initially designated as "to be forgotten" increased more after the second presentation than did those initially designated as "to be remembered." In addition, words not initially recalled were more likely to be recalled after the second presentation if they had been a to-be-forgotten word on the first presentation, relative to being a to-beremembered word on the first presentation. These results were interpreted as due to a release from inhibition.

A final source of support for retrieval inhibition can be found in studies that have used retesting (Reed, 1970; Weiner \& Reed, 1969). Reed (1970, Experiments 3 and 4) presented participants with trigrams that were to be forgotten or to be remembered. After their initial presentation and testing, these items were re-presented as to-be-remembered items and retested. The results showed that, although there was evidence of directed forgetting initially, this effect disappeared on the second testing. Although never using the term inhibition in describing these results, Reed (1970, p. 456) noted that the "first test must therefore have involved a temporary hindrance to retrieval rather than a loss of stored availability in LTM." Related to these findings is evidence that when participants are given a recognition memory test of tobe-remembered items that includes as distractors to-beforgotten items, directed forgetting on a subsequent free recall test does not occur (E. L. Bjork, R. A. Bjork, \& Glenberg, 1973, described in E. L. Bjork, R. A. Bjork, \& Anderson, 1998). Similarly, there is evidence that the manipulations that produce directed forgetting as measured with recall tests are often ineffective when performance is measured with a recognition test (Geiselman et al., 1983; see also Basden et al., 1993). The explanation here is that the appearance of the to-be-forgotten items produces a "release" from retrieval inhibition.

Current interest in the role of retrieval inhibition in human memory includes an expanded scope of application of the concept and concerted efforts to understand its underlying mechanism. The former is exemplified by developmental studies that have attempted to discover age-related differences in retrieval inhibition (Harnishfeger \& Pope, 1996; Zacks, Radvansky, \& Hasher, 1995). The latter includes the study of directed forgetting in the context of implicit memory, which is assumed to exclude the possible role of rehearsal (MacLeod, 1989; E. L. Bjork \& R. A. Bjork, 1996). Considering both the 
importance of the retrieval inhibition concept to understanding memory processing in humans and its applied relevance (e.g., the degree to which it is possible to forget child sexual assault), interest in directed forgetting can be expected to increase.

Despite the popularity of the retrieval inhibition view in human memory, this evidence may not by itself excite interest in adaptive forgetting in animals. One source of inertia might be a comparative bias. Perhaps the kind of retrieval inhibition mechanism inferred to operate in directed forgetting studies is uniquely human. Although the topic of directed forgetting has been studied in animals, this research has tended to emphasize processes that affect encoding, such as rehearsal (Grant, 1998). An even greater concern is that directed forgetting experiments with animals might not involve processes anything like those that have been evoked in the analysis of human directed forgetting (Roper \& Zentall, 1993).

There is another precedent for adaptive forgetting, however, that might be even more appealing to animal researchers than is the topic of motivated forgetting. Anderson and his colleagues (Anderson, 1989, 1990; Anderson \& Milson, 1989; Anderson \& Schooler, 1991) have explicitly discussed memory processing from an evolutionary perspective that emphasizes the adaptive significance of human forgetting. A cogent example of this approach is found in Anderson and Schooler (1991). Their thesis is that the pattern of relationships found between independent variables and memory performance reflect the structure of the environment. Accordingly, "human memory has the form it does because it is adapted to these environmental relationships" (Anderson \& Schooler, 1991, p. 396). More specifically, they argue that the daunting task of managing large collections of memories requires an optimality strategy in which memories more likely to be needed are made more available than memories less likely to be needed. Anderson and Schooler propose that forgetting reflects such an optimality strategy. Among other applications, this approach offers an explanation of the relationships among practice, spacing, and retention interval.

The spacing effect in particular reflects a phenomenon for which the value of the adaptive forgetting perspective can be appreciated. The basic effect is that when human subjects are presented with lists of information, they are less likely to recognize that an item has been repeated when repetitions are spaced than when repetitions occur closely in time. Paradoxically, spaced repetition of items enhances retention of those items (Glenberg, 1979; Melton, 1967). Alternative accounts of the spacing effect have been offered, including explanations that emphasize encoding (Glenberg, 1979). For present purposes, however, it is interesting to note that an adaptive forgetting hypothesis has also been proposed. Cuddy and Jacoby (1982, p. 464) argued that "repeated processing of an item can enhance memory performance but that processing will only be repeated if memory for a prior presentation of an item is not readily accessible." By spacing repetitions, it is more likely that the first occurrence of an item will be forgotten, which in turn will promote additional processing of that item when it is repeated. In this case, initial forgetting of an item enhances subsequent processing of that item, which ultimately improves recall.

The popularity of the adaptive forgetting idea in research on human memory stands in bold contrast to its status in animal memory. Although there have been some attempts to describe adaptive features of animal forgetting (Hendersen, 1985; Kraemer \& Roberts, 1984), the failed process view continues to dominate the analysis of animal memory. For several reasons, it now seems prudent to consider more carefully the possibility that animals also demonstrate adaptive forgetting. One reason to consider adaptive forgetting in animals is that it is relevant to the general objectives of comparative psychology. An important aspect of research on comparative cognition involves knowing the extent to which human memory operates differently from memory in other organisms (Wasserman, 1997). Knowing whether adaptive forgetting is uniquely human is an important question for comparative psychology. Perhaps phenomena such as motivated forgetting reflect mechanisms associated with demands of language processing or the management of large sets of conflicting memories challenges that are less obvious in other animals.

A second consideration is that the existence of adaptive forgetting in animals could provide new research opportunities. The general advantages associated with animal research (e.g., experimental control) could be exploited in the analysis of adaptive forgetting. As a consequence of such research, broader understanding of adaptive forgetting might be realized (examples of which will be discussed later). In addition, research with animals would make it possible to study the neurobiological mechanisms responsible for adaptive forgetting. Animal models have been especially valuable in the study of brain function pertinent to memory processing (Squire, 1992). If adaptive forgetting is functionally distinct from forgetting of the failed process kind, then different neurobiological mechanisms for these two kinds of forgetting will need to be articulated, and research with animals can provide a valuable means to pursue that issue. A related point is that current research with animals on neurobiological foundations of memory is exclusively dominated by the failed process view. If animals do exhibit adaptive forgetting, and if this process of forgetting differs from forgetting of the failed process kind, then it will be necessary to appreciate that distinction in the analysis of brain function. Finally, there has been increased interest in the evolutionary analysis of behavior, both in humans (Buss, 1994) and in other animals (Timberlake, 1994), and the study of adaptive forgetting in animals would be of obvious relevance to that research agenda. 


\section{Conceptual Foundations to Adaptive Forgetting}

The argument that animals exhibit adaptive forgetting begins with some basic conceptual issues. Animal forgetting is usually operationally defined as a relative decrement in performance of a learned response over time (Spear, 1978). The relationship between behavioral phenomena and cognitive constructs is always precarious (Flanagan, 1984), and that relationship can be especially strained by reliance on operational definitions. There is no guarantee that an operational definition will collect behavioral events that are linked to the same type of internal events inferred by a given theory. The critical assumption is that the empirical events to which a theory speaks are indeed of the same kind. If, instead, the definition brings together events that are of a kind only with respect to some arbitrary behavioral protocol, then a specific theory might be inappropriately extended. For example, rigid reliance on an operational definition might preclude analyses that discriminate cases of forgetting with respect to underlying functional processes that distinguish them. Equally unappealing, we might fail to recognize that seemingly disparate behavioral events, some of which do not meet the operational definition in question, depend on a common process. Thus, although operationism imposes conceptual order at the behavioral level, it can also obscure underlying process distinctions that can have profound theoretical significance (Pylyshyn, 1984).

An example of the principle that different processes can lead to the same behavior outcome can be seen in the distinction between decay and retrieval failure theories of forgetting. Each theory ascribes forgetting to a different process: loss of information in the case of decay theory, and inaccessibility to information in the case of retrieval failure theory. Despite the process distinction between them, these two kinds of forgetting are indistinguishable at the behavioral level; both yield a response deficit induced by a retention interval. The central proposition that we now wish to advance is that there may be yet another kind of forgetting expressed by animals: forgetting that is adaptive, which differs from other kinds of forgetting in terms of mechanism and behavioral consequences.

One way to introduce this adaptive kind of forgetting is to contrast animate and inanimate memory systems. Unlike animals, computers are normally immune to forgetting. Once information is stored, forgetting is not an issue with a computer, unless of course a malfunction occurs (i.e., there is a processing failure). It is tempting to conclude from this comparison that biological memory is inherently inferior to computer memory. This prejudice can be strengthened by emphasizing the difference in the representational substrate between the two systems-an emphasis that highlights the greater vulnerability of biological memory to failed processes. Computer memory is based on a physical substrate that changes little over time. In contrast, biological memory depends on a profoundly dynamic substrate. The growth and death of neurons, cellular metabolism, chemical turnover, structural changes in synapses, modification in neuroarchitecture, fluctuations in neurotransmitter levels, and general alterations in physiological status can all contribute to variations in the physical character of an animal's memory. It seems only reasonable, therefore, to expect that these sorts of events will promote forgetting of the failed process kind.

What needs to be appreciated, however, is that not all forgetting is necessarily determined by "hardware"; forgetting can be induced easily in a computer system through appropriate "software." It is a simple matter to arrange relevant components of a computer program to yield input-output relations that would operationally qualify as forgetting. For example, it is possible to change a computer program designed to print an uppercase $A$ on the screen whenever the space bar is pressed to exhibit forgetting; an instruction could be added to the program that cancels the expected A whenever the interval between space-bar inputs exceeds some arbitrary threshold. Clearly, under these conditions, the absence of the expected response would not be attributed to a program malfunction, but rather it would be described as a part of the program. The same principle might also operate in animals, and the idea of "programmed forgetting" can be loosely construed as what is meant by adaptive forgetting.

\section{Adaptive Forgetting in Animals}

The general proposition is that organisms sometimes forget because they are designed to do so, and they may be so designed in order to enhance behavioral plasticity. There are two primary advantages conferred by adaptive forgetting. First, organisms profit from the selective forgetting and remembering of events according to the relevance or biological importance of those events (Kraemer \& Roberts, 1984; Kraemer \& Spear, 1993). Second, adaptive forgetting reflects an efficient and powerful strategy for dealing with conflicting information. Organisms often confront environmental changes that require incompatible behavior; a response that was once appropriate might become inappropriate as the situation changes. These advantages have been cogently summarized by Hendersen (1985):

The real world, in contrast to the laboratory, is inherently unstable, and the longer the time interval between successive contacts with a particular situation, the more likely that the situation will change. Forgetting processes that help map an animal's behavior to the instabilities inherent in a changing world could thus contribute to survival (p. 45).

The language used by Hendersen is conspicuously similar to the discussion of adaptive forgetting in humans presented by Anderson and his colleagues (Anderson, 1989, 1990; Anderson \& Milson, 1989; Anderson \& Schooler, 
1991); both authors emphasize adaptive forgetting as an information management strategy that has evolved in response to demands of a dynamic environment.

The present analysis distinguishes the two advantages of adaptive forgetting in terms of two distinct functions: (1) retrieval suppression of invalid information, and (2) retrieval regulation of competing memories over relatively long retention intervals.

Retrieval suppression involves controlled access to memories that are no longer valid. It is closely related to R. A. Bjork's (1989) concept of retrieval inhibition. A simple example can be used to illustrate the basic idea. Assume that an animal has formed some memory (X) that allows it to behave adaptively in a particular situation. Some signal (A) provides a cue for the retrieval of $X$. Now assume that conditions change so that responding associated with $X$ is no longer appropriate. A new memory that represents the revised situation (Y) must now be encoded. It is possible, however, that $\mathrm{Y}$ is also retrieved by signal $A$, the same cue that previously retrieved X. This is essentially the situation animals face when they begin training on a reversal learning task or when they begin extinction. In order to adapt to this state of affairs, the organism must not only encode $Y$, it must also prevent retrieval of $\mathrm{X}$. Without some kind of retrieval suppression, the organism would be unable to abandon an inappropriate response tendency and adopt a more beneficial reaction. Thus, there must be some mechanism that prevents retrieval of invalid memories so that animals do not perseverate with maladaptive behaviors.

One way to prevent retrieval of an inappropriate memory is to displace it. This was the mechanism adopted by early interference theory as embodied in the principle of unlearning (Melton \& Irwin, 1940). The general idea was that new memories replace older memories with which they conflict. As interference theory evolved, it became obvious that some concept other than displacement was necessary. The phenomenon of proactive interference (PI) and the sometimes-observed recovery of original response tendencies demanded a suppression rather than substitution mechanism (Postman \& Stark, 1969; Postman \& Underwood, 1973). Consistent with human research, the general conclusion from animal studies of PI is that some mechanism like retrieval suppression or inhibition seems to be operating. When a new memory conflicts with a memory that already exists, the original memory becomes inaccessible but remains available (Spear, 1978; Spear \& Riccio, 1994).

Retrieval suppression has a profound advantage over displacement. It allows the organism to profit from the maintenance of memories that become temporarily invalid. Controlled access to existing memories through retrieval suppression obviates the need to reencode memories that lose and then regain validity. In the general example above, if conditions revert so that memory $\mathrm{X}$ is again appropriate, then rapid behavioral adjustment is possible by merely reestablishing retrieval access to $\mathrm{X}$.
In this way, behavioral plasticity is achieved with less effort and more quickly than would be required if conflicting memories were deleted or replaced.

The other form of adaptive forgetting proposed here is retrieval regulation. It is defined as the process whereby relative retrieval access to competing memories changes over a retention interval to promote adaptive behavioral reactions to conflicting experiences. For example, after storing two conflicting memories in succession, an animal might show a retrieval bias for the more recent memory. After a long retention interval, however, the retrieval regulation process might result in either no retrieval bias or a bias for the memory that was first encoded, depending on the behavioral advantages associated with such changes. In most situations, retrieval regulation is preceded by retrieval suppression. As organisms learn competing response tendencies, they initially need to inhibit inappropriate responses, but this behavioral bias often changes over a subsequent retention interval. $\mathrm{Re}$ trieval regulation explains these behavioral changes as instances in which relative retrieval access to competing memories changes in an adaptive manner. More specifically, we will argue that retrieval regulation serves as an alternative to explanations of interference effects that embody the failed process view. Unlike retrieval suppression, retrieval regulation lacks a strong precedent in the human memory literature. Consequently, one of the main contributions of the present analysis is an expansion of the analytical scope of the adaptive forgetting perspective.

We see two primary obstacles to establishing the credibility of adaptive forgetting in animals. First, it is necessary to describe the adaptive nature of phenomena assumed to reflect adaptive forgetting, which can be risky. Identification of phenomena reflecting adaptive forgetting has to include a strong rationale; the mere existence of those phenomena is not by itself sufficient grounds to accept the adaptive nature of those phenomena. Second, it is necessary to move beyond speculation about the adaptiveness of particular instances of forgetting and outline some viable mechanism that could encompass both retrieval suppression and retrieval regulation. Both issues will be addressed here.

\section{Phenomena Reflecting Adaptive Forgetting}

Proactive interference. The general case of PI involves two competing episodes that differ with respect to stimulus-response contingencies but are otherwise equivalent in terms of training parameters and contextual cues. Under these conditions, forgetting of a more recent event $(\mathrm{Y})$ is enhanced by the presence of some prior memory $(\mathrm{X})$. For example, rats trained in a T-maze with black and white arms might first learn to approach the black arm and avoid the white arm (memory X), followed by reversal training in which they learn to approach the white arm and avoid the black arm (memory $Y$ ). Retrieval suppression in this situation would involve 
decreased retrieval access to memory $\mathrm{X}$ during reversal training in order to inhibit choosing the black arm. Retrieval regulation would involve changes in retrieval access to memories $\mathrm{X}$ and $\mathrm{Y}$ over a long retention interval following reversal training. The consequence of these changes would be a decreased tendency to approach the white arm after a retention interval, relative to performance immediately after reversal training (Gleitman, 1971).

An account of this form of PI based on the adaptive forgetting perspective assumes that exposure to conflicting experiences introduces the subject to ambiguity: the world according to $\mathrm{X}$, and the world according to Y. Following acquisition of $\mathrm{Y}$ and after a short retention interval, the impact of ambiguity is minimized by recency. As long as the behavioral consequences associated with retrieval of memory $\mathrm{Y}$ are congruent with its content, the prior validity of $\mathrm{X}$ is irrelevant. The relative validity of memory $\mathrm{Y}$ over memory $\mathrm{X}$ will be preserved as long as memory $Y$ remains valid. This state of affairs changes, however, when there is an extended period of disuse (i.e., a retention interval), during which time neither $Y$ nor $X$ is activated. A relatively long period of disuse alters the relative validity of dormant memories and changes the perspective from which conflicting memories are weighted. In essence, the situation changes to one in which both memories were once valid, although at different points in time, and neither memory has been valid recently (i.e., neither memory has been recently active).

One intelligent reaction to this type of ambiguity is to express behavioral ambivalence: a response pattern in which there is no systematic bias for either one of the two competing behaviors. The principle suggested here is that it is adaptive for an organism to avoid a selective retrieval bias for one of two conflicting memories after a prolonged period of disuse. Following a long retention interval, during which time neither memory has had an impact on behavior, relative recency no longer guarantees the validity of one memory over the other. The adaptive strategy under these conditions is to respond with uncertainty. Perhaps neither episode from the past serves as a reliable indicator of the present, or perhaps one or the other memory is now appropriate. Once a response is made, ambiguity can be resolved, and selective retrieval of the appropriate memory can be established. Until that information is available, however, relative recency alone is not a compelling reason to expect that one memory or the other accurately represents prevailing conditions; however, there may be other factors besides relative recency that could induce such a bias. For example, an asymmetry between the conflicting experiences in terms of the conditions of training (e.g., number or spacing of training trials) could induce a retrieval bias for one memory over the other that would modulate the impact of a retention interval and exaggerate or reverse a response bias that existed prior to the retention interval. It is plausible that when animals are given many more training trials on the second of two conflicting episodes than on the first, retrieval access to the original memory never recovers, and evidence of PI does not appear.

Retrieval regulation in the case of PI reflects a conservative behavioral strategy that has potential long-term benefits and short-term costs. A potential disadvantage of such a process is that, in any one specific case, the most recent of two conflicting memories might still be valid even after a long period of disuse. In the long term, however, retrieval regulation affords an adaptive strategy for managing memories in a dynamic environment. Specifically, it prevents the organism from being unduly tethered to one of multiple response tendencies that were each valid at some point in time. Through behavioral uncertainty, the organism is better positioned to adjust responding to prevailing conditions, as opposed to being strongly committed to one response over another. Thus, rather than viewing PI as a functional failure, adaptive forgetting regards it as a manifestation of a retrieval mechanism that generates behavioral ambivalence, which is an adaptive response strategy when confronting an ambiguous history (see Bolles, 1985, for a seminal discussion of the role of ambiguity in animal learning). The ultimate evolutionary basis for the existence of such a strategy, as Anderson and Schooler (1991) suggest, is that it reflects the exigencies imposed by a dynamic, fluctuating environment.

Extinction and spontaneous recovery. There are two major theoretical approaches to extinction. One view emphasizes memorial content, which assumes that the memory for conditioning is weakened or eliminated by extinction training (e.g., Pearce \& Hall, 1980; Rescorla \& Wagner, 1972). The other approach, which is consistent with an adaptive forgetting framework, adopts a retrieval processing view (see also Bouton 1991; Capaldi, 1966, 1967, 1971; Spear, 1971). This approach has been succinctly summarized by Bolles (1985): "I would suggest as an alternative to the 'loss' theory of extinction, and as a basic working principle, that the animal remembers virtually everything that happens to it in a conditioning experiment" (p. 358). An account of extinction based on an adaptive forgetting perspective assumes that the extinction episode and the original conditioning experience are represented by two independent memories, each associated with a different response tendency. Conditioned responding occurs when the memory for initial conditioning is retrieved, and the absence of a conditioned response, or perhaps some other response, occurs when the memory for extinction is retrieved. At the completion of extinction training, the memory for extinction holds a selective retrieval advantage over the memory for conditioning. After a period of disuse, however, retrieval competition is again evident as expressed by the phenomenon of spontaneous recovery.

Extinction nicely exemplifies the adaptive advantage of retrieval suppression; it makes sense for animals to withhold responses that are no longer associated with expected events (i.e., an unconditioned stimulus [US] in 
the case of Pavlovian conditioning, or a reinforcer in the case of instrumental conditioning). The adaptiveness of spontaneous recovery is less transparent, but there is at least a precedent to view it as an instance of forgetting (Capaldi, 1967; Spear, 1971). As with PI, extinction involves the existence of two conflicting memories: one for original conditioning, and the other for extinction training. Spontaneous recovery can be regarded as another situation in which experience with ambiguous signals renders it adaptive for the animal to express behavioral ambivalence. It is assumed that a period of disuse following extinction reestablishes retrieval access to the conditioning memory. The fact that spontaneous recovery is rarely complete (i.e., responding does not reach the level associated with original conditioning) and that conditioned responding can be quickly suppressed by additional extinction training suggests that the animal is uncertain as to which of the two response tendencies is appropriate, as opposed to the possibility that the memory for the extinction episode has been deleted. If irrelevant memories were simply deleted or replaced, spontaneous recovery and rapid relearning following extinction, which is sometimes observed (Bolles, 1985; Bouton, 1991, 1993), would not occur.

Latent inhibition and release from latent inhibition. Nonreinforced exposure to stimuli prior to conditioning often weakens the expression of acquired responding to these stimuli, an effect termed latent inhibition (LI). For example, if rats are allowed to drink a flavored solution without consequences before they are exposed to that flavor paired with illness, they will exhibit weaker learned aversions to that flavor than they would if the flavor had not been experienced before the conditioning episode. Traditional explanations of LI have almost universally emphasized the acquisition process. It has been assumed that preexposure disrupts encoding of the conditioning episode (Lubow, 1973). There is recent evidence, however, that LI can be reduced or eliminated through reinstatement (e.g., brief presentation of a US just prior to testing; Kasprow, Catterson, Schachtman, \& R. R. Miller, 1984) or by delaying the test episode by several days or weeks (Ackil, Carman, Bakner, \& Riccio, 1992; Bakner, Strohen, Nordeen, \& Riccio, 1991; Kraemer, Randall, \& Carbary, 1991; Kraemer \& Roberts, 1984). These results suggest that LI does not entail a storage deficit. If stimulus preexposure disrupts what an animal subsequently learns about that stimulus, then it should be impossible to eliminate the deficit through events that occur after the learning episode.

An alternative to the storage failure view is the idea that $\mathrm{LI}$ arises from retrieval interference, which makes possible the further conjecture that release from LI reflects adaptive forgetting (see Bouton, 1991, R. R. Miller \& Grahame, 1991, and R. R. Miller, Kasprow, \& Schachtman, 1986, for extended discussions of this approach). As with extinction, LI involves two conflicting memories. In the case of Pavlovian conditioning, the preexpo- sure memory represents the relatively neutral consequences associated with nonreinforced exposure to the conditioned stimulus (CS); the conditioning memory represents the CS-US association. Conditioned responding occurs when the conditioning memory is retrieved, and some other response tendency appears when the preexposure memory is retrieved. The two memories conflict by virtue of their divergent content and associated response tendencies. Thus, retrieval interference is expected when testing occurs soon after conditioning. At this point, retrieval access to the preexposure memory will disrupt selective retrieval of the conditioning memory.

This situation is expected to change, however, over a prolonged retention interval. Another principle needs to be introduced at this point in order to capture the nature of that change. Consistent with the notion that memory processing generally enhances behavioral adaptation, it is reasonable to assume that changes in retrieval access to individual memories will be influenced by the biological significance of those memories: Representational content will impact forgetting (Hendersen, 1985). The idea is that retrieval access to competing memories will change over a period of disuse in such a way that memories of greater importance gain a retrieval advantage over memories of lesser importance. Stated simply, information that is less significant is more likely to undergo forgetting (for examples of this principle, see Aguado, Symonds, \& Hall, 1994, Hendersen, 1985, Kraemer \& Roberts, 1984, and Spear, Hill, \& O'Sullivan, 1965).

A critical feature of this analysis is a distinction between conflicting memories that are symmetrical and those that are asymmetrical with respect to affective and motivational properties. In the case of animal studies of PI, which have used reversal learning to produce conflicting experiences, the competing memories are usually symmetrical with respect to hedonic attributes (Kraemer, 1984; Spear \& Riccio, 1994). For example, rats might be trained to enter the black arm of a T-maze and avoid the white arm by placing food in the black arm; during the second phase of training, the contingencies would be reversed so that food would appear in the white arm instead. Notice that both episodes involve the same hedonic properties: food for entering the correct arm, and the absence of food for entering the incorrect arm. Extinction and LI, in contrast, involve conflicting memories that are hedonically imbalanced. As an example, in Pavlovian conditioning, one episode represents the pairing of a CS with a highly charged affective event (i.e., the US), and the other memory represents the relatively neutral affective consequences associated with mere exposure to the CS.

Our adaptive forgetting analysis of LI assumes that affective attributes of learning episodes influence subsequent retrieval of the memories in which these attributes are represented. Furthermore, the affective asymmetry involved in LI and extinction produces changes in re- 
trieval access that are different from those found with affectively symmetrical memories. Release from LI, as is also true of spontaneous recovery, involves a situation in which access to a more biologically significant memory (e.g., the CS-US association) gains a retrieval advantage over a less significant memory (e.g., a CS-alone episode) over time. The principle suggested here is that it is better to remember an emotionally charged event (e.g., $\mathrm{CS}+\mathrm{US})$ than it is to remember an affectively neutral episode (e.g., CS alone). Release from LI therefore involves a decrease in retrieval access to the older of two competing memories, which is the opposite of what is inferred to occur with PI. This analysis parallels that offered by Hendersen (1985) to account for the finding that rats trained on a fear conditioning task exhibit forgetting of conditioned inhibition (a signal predicts the absence of footshock) but not of conditioned excitation (a signal predicts the occurrence of footshock; see Hendersen, 1978; Thomas, 1979). Again, the idea is that it is better, in the long run, to remember that a signal predicts an aversive event than it is to remember that a signal predicts the absence of an aversive event.

A further implication of the present analysis is that extinction and LI involve a shared process. This possibility has been offered previously by Bouton (1991, 1993). The two phenomena share an undeniable methodological symmetry. In Pavlovian conditioning, both extinction and $\mathrm{LI}$ involve exposing the subject to the CS as well as to CS-US pairings. The only difference between the two procedures is in the order in which these two events occur. It is also the case that neither extinction nor CS preexposure produces conditioned inhibition (Hall \& Minor, 1984; Kaye, Preston, Szabo, Druiff, \& Mackintosh, 1987; Reiss \& Wagner, 1972), and both are context specific (Bouton, 1991; Hall \& Channell, 1986). Despite the procedural and phenomenological similarity between them, extinction and LI have not received parallel theoretical treatment. In contrast to that trend, the present position is that extinction and LI involve a common process: one that implicates retrieval rather than storage operations, and one that reflects adaptive forgetting.

\section{Retrievability: A Mechanism for Adaptive Forgetting}

The argument for adaptive forgetting must include more than speculation on the adaptiveness of behavioral phenomena. The question of mechanism must also be considered. Specifically, it is necessary to propose a mechanism that encompasses both forms of adaptive forgetting proposed here: response suppression and retrieval regulation. The concept of retrievability will be introduced for that purpose.

Retrievability is best understood within the context of an activation view of memory retrieval. Activation models assume that memories are retrieved when a sufficient number or type of memory attributes is activated by appropriate stimulus events (Bower, 1967; Spear, 1973 ;
Underwood, 1969). Accordingly, retrievability can be defined as some threshold value of attribute activation that must be reached in order for a memory to be retrieved. The most important assumption added here is that retrievability can vary. When retrievability is increased, a memory becomes more accessible; when it is decreased, a memory becomes less accessible. By postulating a set of simple rules that govern how and when retrievability changes, Kraemer and Spear (1993) offered a preliminary retrieval-based model that captures the essence of an adaptive forgetting approach.

The first rule of this model concerns retrieval suppression. It states that an inconsistency between the content of a target memory and consequences associated with its retrieval will decrease the retrievability of that memory. For example, the absence of an expected US or reinforcer, as occurs early during reverse discrimination training and extinction, will gradually decrease the retrievability of the original memory to a level at which it is no longer activated by formerly effective retrieval cues.

The remaining rules are pertinent to retrieval regulation. These rules are designed to explain changes in retrieval access to conflicting memories that occur as a function of disuse (i.e., a retention interval). The first of these rules emphasizes a distinction between memories that are affectively symmetrical and those that are affectively asymmetrical. In the case of the former, the rule is that the retrievability of two conflicting memories will tend toward parity over a retention interval. There will be a decrease in the relative retrievability of the more recent of the two memories and an increase in the relative retrievability of the older of the two memories over a period of disuse. The consequence of these changes is PI; subjects respond in accord with the more recent memory less strongly than they did before the retention interval.

There is an important feature of the retrievability view of PI that needs to be emphasized. It is possible for PI to appear without any discernible recovery of initial responding. The retrieval regulation principle implies that responses appropriate to the second of two conflicting learning episodes can diminish after a retention interval, without evidence that responses appropriate to the first episode have recovered. This assumption circumvents a difficulty encountered by the response competition principle of early interference theory. Initial evidence that forgetting could be induced through interference from prior learning was the finding that when participants expressed forgetting of a target task that was preceded by some other learning episode, they often expressed responses appropriate to the episode that had preceded the target task. For example, in a paired associate learning task, participants given $d o g$ paired with tree on one list and then $d o g$ paired with house on a second list might provide "tree" as a response when tested with the stimulus item dog. It has also been shown, however, that PI sometimes appears despite no evidence that initial response tendencies have recovered. (Postman \& Stark, 
1969; Postman \& Underwood, 1973). The retrievability approach obviates this concern by assuming that the source of PI is independent of response competition.

A different rule applies for cases in which conflicting memories are affectively asymmetrical, as is true with extinction and LI. A retention interval following either extinction training or a conditioning episode that was preceded by stimulus preexposure will decrease the relative retrieval access to the less emotionally charged memory. This process can be achieved either by a decrease in the retrievability of the less emotionally charged memory (e.g., the memory for a CS-alone episode) or by an increase in the retrievability of the more emotionally charged memory (e.g., a CS-US memory). In either way, the result will be a selective retrieval advantage for the more emotionally charged memory following a retention interval.

This processing rule can also be extended to the finding that response inhibition is forgotten more rapidly than is response excitation (Hendersen, 1978) and to evidence that memory for nonreward is forgotten more readily than is memory for reward (Spear, 1967; Spear et al., 1965; Spear \& Spitzner, 1967). Both of these situations embody the principle that events of greater biological importance are remembered better than are events of lesser biological importance, which reflects adaptive forgetting. Thus, even in the absence of a conflicting memory, the retrievability of an affectively neutral memory can decline (Aguado et al., 1994; Kraemer \& Roberts, 1984). In contrast, the retrievability of an affectively charged memory might remain unchanged or might even increase over a retention interval (e.g., Batsel \& Best, 1992, 1994). This assumption is supported by considerable evidence that simple learning episodes (e.g., exposure to a light paired with footshock) are impressively well retained over very long retention intervals (Spear, 1978).

The final rule described by Kraemer and Spear (1993) is used to account for the pervasive phenomenon of reinstatement. In a variety of circumstances, it is possible to mitigate or eliminate forgetting by exposing the subject to relevant aspects of the learning episode prior to testing. One version of this phenomenon is the warm-up effect. Brief exposure to the test context can alleviate forgetting that would otherwise obtain (Spear, 1973). Another version of reinstatement involves exposure to a US after extinction training or after conditioning with a preexposed CS (LI). Brief pretest presentations of the US are sufficient to enhance conditioned responding that would be otherwise weak or nonexistent (Bouton, 1991; R. R. Miller et al., 1986). The processing rule in these situations is that exposure to information represented by a memory can increase the subsequent retrievability of that memory, even when such exposure by itself is insufficient for retrieval. Thus, although presentation of a US just prior to testing might not retrieve a CS-US memory, it can make subsequent retrieval of that memory more likely.

\section{Summary and Implications}

The main purpose of the present argument is to initiate discussion about the possibility that some forgetting in animals is adaptive. A secondary objective is to outline a retrieval-based model that could potentially account for instances of adaptive forgetting in animals and humans. Each of these objectives has important implications.

With respect to greater interest in adaptive forgetting in animals, one implication is that it would promote further interactions between human memory and animal memory research. As described above, there has been considerable attention given to adaptive forgetting in the study of human memory, but parallel enthusiasm for the idea is yet to emerge in the study of animal memory. Comparative interest in adaptive forgetting could initiate collaboration between human and animal memory researchers that would expand and enrich the analytical scope of research on adaptive forgetting. For example, if there exists an animal analog to retrieval inhibition (R. A. Bjork, 1989), then it will be possible to extend the analysis of this process by exploiting the advantages associated with research with animals.

Greater interest in adaptive forgetting in animals also has implications for the neurobiology of memory. A substantial component of research in this area depends on animal models. That research is currently dominated by the failed process view. This bias can be partially understood as a natural consequence of methodology; the invasive techniques on which much of neurobiology depends evoke the failed process perspective. For example, the kinds of brain-behavior relations used to generate inferences about memory processing usually entail behavioral dysfunctions. Reliance on a strategy of disruptive intervention can lead naturally to the overgeneralization that all forgetting corresponds to memory dysfunction. If, however, animals express adaptive forgetting and if that kind of forgetting entails a mechanism different from that involved in forgetting of the failed process kind, then it will be necessary to extend that distinction to neurobiological analyses. The difficult challenge here will be to identify the criteria by which adaptive forgetting can be distinguished from the failed process kind of forgetting at the behavior or cognitive level.

The retrievability mechanism itself has implications for memory research that are independent of its connection to adaptive forgetting. For example, the modest retrieval model outlined here makes possible a common process explanation for phenomena that are typically segregated: general forgetting, acquisition of reversal learning, PI, extinction, spontaneous recovery, LI, release from $\mathrm{LI}$, and reinstatement effects. This approach can perhaps be further extended to encompass an even broader range of phenomena, including context effects on retention (Riccio, Richardson, \& Ebner, 1984), forgetting of stimulus attributes (Riccio, Ackil, \& BurchVernon, 1992), blocking (Batsell \& Best, 1992; J. S. Miller, Jagielo, \& Spear, 1990; J. S. Miller, McKinzie, 
Kraebel, \& Spear, 1996), and overshadowing (Kraemer, Lariviere, \& Spear, 1988). Alternative theoretical approaches to learning have not attempted to capture this diverse range of phenomena within the same basic explanatory context (Grossberg, 1982; Holyoak, Koh, \& Nisbett, 1989; Mackintosh, 1975; Rescorla \& Wagner, 1972; Wagner, 1981). In general, there has been reluctance to advance theoretical accounts of acquired behavior that integrate acquisition and retention phenomena (cf. Capaldi, 1971; Spear, 1981). In contrast, the present approach, as is true of other retrieval based analyses (e.g., Bouton, 1993; R. R. Miller et al., 1986; Spear, 1981), assumes that retrieval is as much involved in acquisition as it is in retention. As learning progresses, target memories become more easily retrieved, so that the behavioral changes apparent in a learning curve reflect improved retrieval access to target memories as well as the progressive storage of those memories.

Finally, the retrievability principle also provides a potential mechanism with which to explain the interface between cognitive and emotive processes. For example, one way in which the hedonic properties of learning episodes can influence memory processing is in terms of the degree to which these attributes alter accessibility to stored representations. The retrievability construct can be used to explain both memory enhancement and forgetting of episodes that include significant emotional attributes. Affective attributes of learning episodes might not just be incorporated into the contents of memories; these features might also influence retrieval of those memories and might do so in an adaptive fashion. This kind of theoretical emphasis on interrelationships between cognitive and emotive factors has not been sufficiently considered in recent animal memory research, despite a growing emphasis on such interrelationships in the study of human memory (Bower, 1981).

\section{REFERENCES}

Ackil, J. K., Carman, H. M., Bakner, L., \& Riccio, D. C. (1992). Reinstatement of latent inhibition following a reminder treatment in a conditioned taste aversion paradigm. Behavioral \& Neural Biology, 58, 232-235.

Aguado, L., Symonds, M., \& Hall, G. (1994). Interval between preexposure and test determines the magnitude of latent inhibition: Implications for an interference account. Animal Learning \& Behavior, 22, 188-194.

American Psychological Association (1995). Working group on in vestigation of memories of childhood abuse: Final report. Washington, DC: Author.

ANDERSON, J. R. (1989). A rational analysis of human memory. In H. L. Roediger III \& F. I. M. Craik (Eds.), Varieties of memory and consciousness: Essays in honour of Endel Tulving (pp. 195-210). Hillsdale, NJ: Erlbaum.

ANDERSON, J. R. (1990). The adaptive character of thought. Hillsdale, $\mathrm{NJ}$ : Erlbaum.

Anderson, J. R., \& Milson, J. R. (1989). Human memory: An adaptive perspective. Psychological Review, 96, 703-719.

Anderson, J. R., \& Schooler, L. J. (1991). Reflections of the environment in memory. Psychological Science, 2, 396-408.

Bakner, L., Strohen, K., Nordeen, M., \& Riccio, D. C. (1991). Postconditioning recovery from the latent inhibition effect in conditioned taste aversion. Physiology \& Behavior, 50, 1269-1272.

Basden, B. H., Basden, D. R., \& Gargano, G. J. (1993). Directed for- getting in implicit and explicit memory tests: A comparison of methods. Journal of Experimental Psychology: Learning, Memory, \& Cognition, 19, 603-616.

BATSELl, W. R., JR., \& Best, M. R. (1992). Variations in the retention of taste aversions: Evidence for retrieval competition. Animal Learning \& Behavior, 20, 146-159.

BATSELl, W. R., JR., \& BesT, M. R. (1994). The role of US novelty in retention interval effects in single-element taste-aversion learning. Animal Learning \& Behavior, 22, 332-340.

Bechtel, W. (1988). Philosophy of science. Hillsdale, NJ: Erlbaum. BJork, E. L., \& BJork, R. A. (1996). Continuing influences of to-beforgotten information. Consciousness \& Cognition, 5, 176-196.

BJork, E. L., BJork, R. A., \& ANDERson, M. C. (1998). Varieties of goal-directed forgetting. In J. M. Golding \& C. M. MacLeod (Eds.), Intentional forgetting: Interdisciplinary approaches (pp. 103-137). Mahwah, NJ: Erlbaum.

BjoRK, R. A. (1972). Theoretical implications of directed forgetting. In A. W. Melton \& E. Martin (Eds.), Coding processes in human memory (pp. 217-235). Washington, DC: Winston.

BJORK, R. A. (1989). Retrieval inhibition as an adaptive mechanism in human memory. In H. L. Roediger III \& F. I. M. Craik (Eds.), Varieties of memory and consciousness: Essays in honour of Endel Tulving (pp. 309-330). Hillsdale, NJ: Erlbaum.

BJORK, R. A. (1998). Intentional forgetting in perspective: Comments, conjectures, and some directed remembering. In J. M. Golding \& C. M. MacLeod (Eds.), Intentional forgetting: Interdisciplinary approaches (pp. 453-481). Mahwah, NJ: Erlbaum.

Bolles, R. C. (1985). A cognitive, nonassociative view of inhibition. In R. R. Miller \& N. E. Spear (Eds.), Information processing in animals: Conditioned inhibition (pp. 355-367). Hillsdale, NJ: Erlbaum.

Bouton, M. E. (1991). Context and retrieval in extinction and in other examples of interference in simple associative learning. In L. W. Dachowski \& C. F. Flaherty (Eds.), Current topics in animal learning: Brain, emotion, and cognition (pp. 25-53). Hillsdale, NJ; Erlbaum.

Bouton, M. E. (1993). Context, time, and memory retrieval in the interference paradigms of Pavlovian conditioning. Psychological Bulletin, 114, 80-99.

BOWER, G. H. (1967). A multi-component theory of the memory trace. In K. W. Spence \& J. T. Spence (Eds.), The psychology of learning and motivation (Vol. 1, pp. 229-325). New York: Academic Press.

Bower, G. H. (1981). Mood and memory. American Psychologist, 36, 129-148.

Buss, D. M. (1994). The evolution of desire. New York: Harper Collins. CAPALDI, E. J. (1966). Partial reinforcement: A hypothesis of sequential effects. Psychological Review, 73, 459-477.

CAPALDI, E. J. (1967). A sequential hypothesis of instrumental learning. In K. W. Spence \& J. T. Spence (Eds.), The psychology of learning and motivation (Vol. 1, pp. 67-156). New York: Academic Press.

CAPALDI, E. J. (1971). Memory and learning: A sequential viewpoint. In W. K. Honig \& P. H. R. James (Eds.), Animal memory (pp. $115-$ 154). New York: Academic Press.

Cuddy, L. J., \& JACOBY, L. L. (1982). When forgetting helps memory: An analysis of repetition effects. Journal of Verbal Learning \& Verbal Behavior, 21, 451-467.

EPSTEIN, W. (1972). Mechanisms of directed forgetting. In G. H. Bower (Ed.), The psychology of learning and motivation (Vol. 6, pp. 147191). New York: Academic Press.

Flanagan, O. J. (1984). The science of the mind. Cambridge, MA: MIT Press.

FrEUD, S. (1962). Introductory lectures on psychoanalysis. In J. Strachey (Ed. \& Trans.), The standard edition of the complete psychological works of Sigmund Freud (Vol. 6). London: Hogarth Press. (Original work published 1920)

FREYd, J. J. (1996). Betrayal trauma. Cambridge, MA: Harvard University Press.

Geiselman, R. E., \& Bagheri, B. (1985). Repetition effects in directed forgetting: Evidence for retrieval inhibition. Memory \& Cognition, 13, 57-62.

Geiselman, R. E., Bjork, R. A., \& Fishman, D. L. (1983). Disrupted retrieval in directed forgetting: A link with posthypnotic amnesia. Journal of Experimental Psychology: General, 112, 58-72.

Gleitman, H. (1971). Forgetting of long-term memories in animais. In 
W. K. Honig \& P. H. R. James (Eds.), Animal memory (pp. 1-44). New York: Academic Press.

GLENBERG, A. M. (1979). Component-levels theory of the effects of spacing of repetitions on recall and recognition. Memory \& Cognition, 7, 95-112.

GolDING, J. M., \& LoNG, D. L. (1998). There's more to intentional forgetting than directed forgetting: An integrative review. In J. M. Golding \& C. M. MacLeod (Eds.), Intentional forgetting: Interdisciplinary approaches (pp. 59-102). Mahwah, $\mathrm{NJ}$ : Erlbaum.

GRaNT, D. S. (1998). Directed forgetting. In J. M. Golding \& C. M. MacLeod (Eds.), Intentional forgetting: Interdisciplinary approaches (pp. 239-264). Mahwah, NJ: Erlbaum.

Grossberg, S. (1982). Processing of expected and unexpected events during conditioning and attention: A psychophysiological theory. Psychological Review, 89, 529-572.

Hall, G., \& Channell, S. (1986). Context specificity of latent inhibition in taste aversion learning. Quarterly Journal of Experimental Psychology, 38B, 121-139.

HaLL, G., \& MiNOR, H. (1984). A search for context-stimulus associations in latent inhibition. Quarterly Journal of Experimental Psychology, 36B, 145-169.

HARNiShFEGER, K. K., \& POPE, R. S. (1996). Intending to forget: The development of cognitive inhibition in directed forgetting. Journal of Experimental Child Psychology, 62, 292-315.

HENDERSEN, R. W. (1978). Forgetting of conditioned fear inhibition. Learning \& Motivation, 9, 16-30.

Hendersen, R. W. (1985). Fearful memories: The motivational significance of forgetting. In F. R. Brush \& J. B. Overmier (Eds.), Affect, conditioning, and cognition: Essays on the determinants of behavior (pp. 43-53). Hillsdale, NJ: Erlbaum.

HolyoAK, K. J., КoH, K., \& NisbeTt, R. E. (1989). A theory of conditioning: Inductive learning within rule-based default hierarchies Psychological Review, 96, 315-340.

JAMES, W. (1890). The principles of psychology. Cambridge, MA: Harvard University Press.

JoHNSON, H. (1994). Processes of successful intentional forgetting. Psychological Bulletin, 116, 274-292.

Kasprow, W. J., Catterson, D., Schachtman, T. R., \& Miller, R. R. (1984). Attenuation of latent inhibition by post-acquisition reminder Quarterly Journal of Experimental Psychology, 36B, 53-63.

Kaye, H., Preston, G., Szabo, L., Druiff, H., \& Mackintosh, N. J. (1987). Context specificity of conditioning and latent inhibition: Evidence for a dissociation of latent inhibition and associative interference. Quarterly Journal of Experimental Psychology, 39B, 127-145.

KRAEMER, P. J. (1984). Forgetting of visual discriminations by pigeons. Journal of Experimental Psychology: Animal Behavior Processes, $10,530-542$.

Kraemer, P. J., Lariviere, N. A., \& Spear, N. E. (1988). Expression of a taste aversion conditioned with an odor-taste compound: Overshadowing is relatively weak in weanlings and decreases over a retention interval in adults. Animal Learning \& Behavior, 16, 164-168

Kraemer, P. J., Randall, C. K., \& Carbary, T. J. (1991). Release from latent inhibition with delayed testing. Animal Learning \& Behavior 19, 139-145.

Kraemer, P. J., \& Roberts, W. A. (1984). The influence of flavor preexposure and test interval on conditioned taste aversions in the rat. Learning \& Motivation, 15, 259-278.

KRAEMER, P. J., \& SPEAR, N. E. (1993). Retrieval processes and conditioning. In T. Zentall (Ed.), Animal cognition (pp. 87-107). Hillsdale, $\mathrm{NJ}$ : Erlbaum.

Loftus, E. F. (1993). The reality of repressed memories. American Psychologist, 48, 518-537.

Lubow, R. R. (1973). Latent inhibition. Psychological Bulletin, 79, 398-407.

Mackintosh, N. J. (1975). A theory of attention: Variations in the associability of stimuli with reinforcement. Psychological Review, 82, 276-298.

MACLEOD, C. M. (1989). Directed forgetting affects both direct and in- direct tests of memory. Journal of Experimental Psychology: Learning. Memory, \& Cognition, 15, 13-21.

MACLEOD, C. M. (1998). Directed forgetting. In J. M. Golding \& C. M. MacLeod (Eds.), Intentional forgetting: Interdisciplinary approaches (pp. 1-57). Mahwah, NJ: Erlbaum.

Maslow, A. H. (1966). The psychology of science. New York: Harper \& Row.

MeLton, A. W. (1967). Repetition and retrieval from memory. Science, 158, 532 .

Melton, A. W., \& Irwin, J. M. (1940). The influence of degree of interpolated learning on retroactive inhibition and the overt transfer of specific responses. American Journal of Psychology, 53, 173-203.

Miller, J. S., JaGielo, J. A., \& SPEAR, N. E. (1990). Changes in the retrievability of associations to elements of the compound CS determine the expression of overshadowing. Animal Learning \& Behavior, 18, $157-161$.

Miller, J. S., McKinzie, D. L., Kraebel, K. S., \& Spear, N. E. (1996). Changes in the expression of stimulus selection: Blocking represents selective memory retrieval rather than selective associations. Learning \& Motivation, 27, 307-316.

MilleR, R. R., \& Grahame, N. J. (1991). Expression of learning. In L.W. Dachowski \& C. F. Flaherty (Eds.), Current topics in animal learning: Brain, emotion, and cognition (pp. 95-117) Hillsdale, NJ: Erlbaum.

Miller, R. R., KaSPROW, W. J., \& SCHAChtMAN, T. R. (1986). Retrieval variability: Sources and consequences. American Journal of Psychology, 99, 145-218.

Pearce, J. M., \& Hall, G. (1980). A model for Pavlovian learning: Variations in the effectiveness of conditioned but not of unconditioned stimuli. Psychological Review, 87, 532-552.

Postman, L., \& STARK, K. (1969). Role of response availability in transfer and interference. Journal of Experimental Psychology, 79, $168-177$

Postman, L., \& Underwood, B. J. (1973). Critical issues in interference theory. Memory \& Cognition, 1, 19-40.

Pylyshyn, Z. W. (1984). Computation and cognition. Cambridge, MA: MIT Press.

REED, H. (1970). Studies of the interference process in short-term memory. Journal of Experimental Psychology, 84, 452-457.

REISS, S., \& WAGNER, A. R. (1972). CS habituation produces a "latent inhibition effect" but no active "conditioned inhibition." Learning \& Motivation, 4, 237-245.

ReSCORLA, R. A., \& WAGNeR, A. R. (1972). A theory of Pavlovian conditioning: Variations in the effectiveness of reinforcement and nonreinforcement. In A. H. Black \& W. F. Prokasy (Ed.), Classical conditioning II: Current research and theory (pp.64-99). New York: Appleton-Century-Crofts.

Riвot, T. A. (1882). Diseases of memory: An essay in the positive psychology. New York: Appleton-Century-Crofts.

Riccio, D. C., ACKIL, J., \& BurCh-Vernon, A. (1992). Forgetting of stimulus attributes: Methodological implications for assessing associative phenomena. Psychological Bulletin, 112, 433-445.

Riccio, D. C., Richardson, R., \& Ebner, D. L. (1984). Memory retrieval deficits based upon altered contextual cues: A paradox. Psychological Bulletin, 96, 152-165.

ROPER, K. L., \& ZENTALL, T. R. (1993). Directed forgetting in animals. Psychological Bulletin, 113, 513-532.

SPEAR, N. E. (1967). Retention of reinforcer magnitude. Psychological Review, 64, 216-234.

Spear, N. E. (1971). Forgetting as retrieval failure. In W. K. Honig \& P. H. R. James (Eds.), Animal memory (pp. 45-109). New York: Academic Press.

SPEAR, N. E. (1973). Retrieval of memory in animals. Psychological Review, 80, 163-194.

SPEAR, N. E. (1978). The processing of memories: Forgetting and retention. Hillsdale, NJ: Erlbaum.

SPEAR, N. E. (1981). Extending the domain of memory retrieval. In N. E. Spear \& R. R. Miller (Eds.), Information processing in animals. Memory mechanisms (pp. 341-378). Hillsdale, NJ: Erlbaum. 
Spear, N. E., Hill, W. F., \& O’Sullivan, D. J. (1965). Acquisition and extinction after initial trials without reward. Journal of Experimental Psychology, 69, 25-29.

SPEAR, N. E., \& RICCIO, D. C. (1994). Memory: Phenomena and principles. Boston: Allyn \& Bacon.

SPEAR, N. E., \& SPITZNER, J. H. (1967). Effect of initial nonrewarded trials: Factors responsible for increased resistance to extinction. Journal of Experimental Psychology, 74, 525-537.

SQUIRE, L. R. (1992). Memory and the hippocampus: A synthesis from findings with rats, monkeys, and humans. Psychological Review, 99 195-231.

Thомаs, D. (1979). Retention of conditioned inhibition in a bar-press suppression paradigm. Learning \& Motivation, 10, 161-177.

TimberLAKE, W. (1994). Behavior systems, associationism, and Pavlovian conditioning. Psychonomic Bulletin \& Review, 1, 405-420

UNDERWOOD, B. J. (1969). Attributes of memory. Psychological Review, 76, 559-573.
WAGNER, A. R. (1981). SOP: A model of automatic memory processing in animal behavior. In N. E. Spear \& R. R. Miller (Eds.), Information processing in animals: Memory mechanisms (pp. 5-47). Hillsdale, NJ: Erlbaum.

Wasserman, E. A. (1997). The science of animal cognition: Past, present, and future. Journal of Experimental Psychology: Animal Behavior Processes, 23, 123-135.

WEINER, B., \& REED, H. (1969). Effects of the instructional sets to remember and forget on short-term retention: Studies of rehearsal control and retrieval inhibition (repression). Journal of Experimental Psychology, 79, 226-232.

Zacks, R. T., Radvansky, G., \& Hasher, L. (1995). Studies of directed forgetting in older adults. Journal of Experimental Psychology: Learning, Memory, \& Cognition, 32, 143-156.

(Manuscript received December 24, 1996; revision accepted for publication March 18, 1997.) 